\title{
A SIMPLE ALGORITHM FOR COMPUTING SEVERAL SEQUENCES SYNTHESIS
}

Wang Mingsheng, Qi sihan, Feng denguo

Engineering Research Center for Information Security Technology

Institute of Software, Chinese Academy of Sciences

Beijing 100080, Beijing P.O. Box 8718, P.R. China

wangmsh@ercist.iscas.ac.cn

Abstract In this paper, we use module Grobner bases to Compute several sequences synthesis problem. It is a natual generalization of Fitzpatrick's algorithm.

Keywords: Module Grobner bases, Several sequences synthesis, Buchberger algoruthm.

\section{Introduction}

Sevaral sequences synthesis problem have been considered by many authors, for example [1], [2], [10]. In [1], [2], the authors considered this problem using Grobner bases method of ideal and their methods involved in solving equations in at least 2 variables. In [9], the author discovered a new approach to solve key equation by using Grobner bases method of submodule of free module over polynomial ring in one variable. In this paper, we extend the method of [9] to the general case.

Let $F$ be a field and $F[x]$ be a polynomial ring in variable $x$. Let $s_{1}=\left(s_{01}, s_{11}, \cdots, s_{n_{1} 1}\right)$,

$s_{2}=\left(s_{02}, s_{12}, \cdots, s_{n_{2} 2}\right), \cdots, s_{t}=\left(s_{0 t}, s_{1 t}, \cdots, s_{n_{t} t}\right)$ be $t$ linear recurring sequences of finite length in $F$. A linear feedback shift register of length $l$ with feedback coeficients $\left(\sigma_{1}, \sigma_{2}, \cdots, \sigma_{l}\right)$ is said to generate $s_{1}, \cdots, s_{t}$, if

$$
s_{j i}=\sum_{r=1}^{l} \sigma_{r} s_{j-r i}, \quad i=1, \cdots, t, \quad 1 \leq j \leq l
$$

An important problem in the theory of coding and cryptography is the synthesis problem:

The original version of this chapter was revised: The copyright line was incorrect. This has been corrected. The Erratum to this chapter is available at DOI: 10.1007/978-0-387-35515-3_53 
Given several sequences in $F$ of finite but possibly different length, find the linear feedback shift register of least length which generate all the sequences.

We know that the synthesis problem have been studied by many authors, but as far as we know, we don't still see methods that use module Grobner bases. In this paper, we give a very natural approach to this problem. Actually, we only need to extend the method of [9] to general case.

\section{REVIEW OF MODULE GROBNER BASES}

Grobner bases theory for free module of finite rank over the polynomial ring is a basic tool in many applications. Its complete discusions may be found in [3], [4], [5], [6], [7].

Let $R$ be a polynomial ring $F\left[x_{1}, \cdots, x_{n}\right]$ and $R^{m}$ be a free module of rank $m$. For $\mathbf{f}=\left(f_{1}, \cdots, f_{m}\right) \in R^{m}, \mathbf{f}=f_{1} e_{1}+\cdots+f_{m} e_{m}$, where $\left\{e_{1}=\right.$ $\left.(1,0, \cdots, 0), e_{2}=(0,1,0, \cdots, 0), \cdots, e_{m}=(0, \cdots, 0,1)\right\}$ is a standard basis of $R^{m}$.

For $\alpha=\left(\alpha_{1}, \cdots, \alpha_{n}\right) \in \mathbb{N}$, we set $x^{\alpha}=x_{1}^{\alpha_{1}} x_{2}^{\alpha_{2}} \cdots x_{n}^{\alpha_{n}}$. A monomial $\mathbf{m}$ in $R$ is an element of the form $x^{\alpha} e_{i}$ for some $e_{i}$. In this case, we say $\mathrm{m}$ contains the standard basis vector $e_{i}$. every element of $\mathrm{f} \in R$ can be written in a unique way as a $F$ - linear combination of monomials $\mathbf{m}_{\mathbf{i}}$

$$
\mathbf{f}=\sum_{i=1}^{n} c_{i} \mathbf{m}_{\mathbf{i}} .
$$

The product $c \cdot \mathbf{m}$ of a monomial $\mathbf{m}$ with an element $c \in F$ is called a term and $c$ is called its coefficient.

If $\mathbf{m}_{\mathbf{1}}=x^{\alpha_{1}} e_{i}, \mathbf{m}_{\mathbf{2}}=x^{\alpha_{2}} e_{j}$ are monomials, then we say that $\mathbf{m}_{\mathbf{1}}$ divides $\mathbf{m}_{\mathbf{2}}$ if and only if $i=j$ and $x^{\alpha_{1}}$ divides $x^{\alpha_{2}}$. If $\mathbf{m}_{\mathbf{1}}$ divides $\mathbf{m}_{\mathbf{2}}$, we define the quotient $\frac{\mathbf{m}_{2}}{\mathbf{m}_{1}}$ to $\frac{x^{\alpha_{2}}}{x^{\alpha_{1}}}$. In general, we define $\operatorname{LCM}\left(\mathbf{m}_{\mathbf{1}}, \mathbf{m}_{\mathbf{2}}\right)=$ $\begin{cases}\operatorname{LCM}\left(x^{\alpha_{1}}, x^{\alpha_{2}}\right) & \text { if } i=j \\ 0 & \text { otherwise }\end{cases}$

We say that a total order $>$ on the monomials of $R^{m}$ is monomial order (or term order) if

(1) for every pair of monomials $\mathbf{m}_{\mathbf{1}}, \mathbf{m}_{\mathbf{2}}$, if $\mathbf{m}_{\mathbf{1}}>\mathbf{m}_{\mathbf{2}}$, then $x^{\alpha} \mathbf{m}_{\mathbf{1}}>$ $x^{\alpha} \mathbf{m}_{\mathbf{2}}$ for every monomial $x^{\alpha} \in R$, and

(2) for any monomial $\mathbf{m} \in R^{m}$ and $x^{\alpha} \in R$, if $x^{\alpha} \neq 1$. then $x^{\alpha} \mathbf{m}>\mathbf{m}$. Given a monomial order $>$ on monomials, we can write any element $\mathrm{f} \in R^{m}$ as a sum of terms

$$
\mathbf{f}=\sum_{i=1}^{t} c_{i} \mathbf{m}_{\mathbf{i}}
$$


with $c_{i} \neq 0$ and $\mathbf{m}_{1}>\cdots>\mathbf{m}_{\mathbf{t}}$. We define the leading coefficient, leading monomial, and leading term of $f$ as follows:

$$
\begin{gathered}
\mathrm{LC}_{>}(\mathrm{f})=\mathrm{c}_{1} \\
\mathrm{LT}_{>}(\mathrm{f})=\mathrm{m}_{1} \\
\mathrm{LM}_{>}(\mathrm{f})=\mathrm{c}_{1} \mathrm{~m}_{1} .
\end{gathered}
$$

Given a monomial order in $R^{m}$, we can divide by a set $G$ of $R^{m}$. For details, we refer to [6], [7].

Division algorithm in $R^{m}$. Fix a monomial order $>$ on $R^{m}$ and let $G=\left(\mathrm{g}_{1}, \cdots, \mathrm{g}_{\mathrm{s}}\right)$ be an order $s$-tuple of elements of $R^{m}$. Then every $\mathrm{g} \in R^{m}$ can be written as

$$
\mathrm{g}=b_{1} \mathbf{g}_{1}+\cdots+b_{s} \mathbf{g}_{\mathbf{s}}+\mathbf{r}
$$

where $b_{i} \in R, \mathbf{r} \in R^{m}, \mathrm{LT}\left(\mathrm{b}_{\mathrm{i}} \mathrm{g}_{\mathbf{i}}\right) \leq \mathrm{LT}(\mathrm{g})$ for all $i$, and either $\mathbf{r}=0$, $\mathbf{r}$ is a $F$ - linear combination of monomials none of which is divisible by any of $\operatorname{LT}\left(\mathrm{g}_{1}\right), \cdots, \mathrm{LT}\left(\mathrm{g}_{\mathrm{s}}\right)$. We call $\mathbf{r}$ the remainder on division by $G$ and denote it by $\bar{g}^{G}$.

Now, we give the definition of Grobner bases.

Definition 2.1 Let $M$ be a submodule of $R^{m}$ and $>$ be a monomial order.

A finite set $G=\left\{g_{1}, \cdots, g_{s}\right\}$ is called a Grobner bases for $M$ if $<$ $\operatorname{LT}(\mathrm{M})>=<\operatorname{LT}\left(\mathrm{g}_{1}\right), \cdots, \operatorname{LT}\left(\mathrm{g}_{\mathrm{s}}\right)>$, where the symbol $\mathrm{LT}(\mathrm{S})$ denotes the set of leading terms of $S$, and $\langle S\rangle$ denotes the submodule of $R^{m}$ generated by $S$.

Let $G$ be a Grobner bases for $M$, if $\forall g_{\mathbf{i}}, \mathrm{LC}\left(\mathrm{g}_{\mathbf{i}}\right)=1$, and $\mathrm{LT}\left(\mathrm{g}_{\mathbf{i}}\right) \notin<$ $\mathrm{LT}\left(\mathrm{G} \backslash \mathrm{g}_{\mathbf{i}}\right)>$, then we say that $G$ is a minimal Grobner bases for $M$.

Once we have a monomial order $>$, for every submodule $M$ of $R^{m}$, there is a Grobner bases for $M$ with respect to $>$. If we have a group of generators of $M$, then we can find a Grobner bases for $M$ by Buchberger algorithm. For details, we refer to [6], [7].

\section{COMPUTATIONAL METHODS}

Let $\sigma(x)=1+\sigma_{1} x+\sigma_{2} x^{2}+\cdots+\sigma_{l} x^{l}$ and $S_{i}=s_{0 i}+s_{1 i} x+s_{2 i} x^{2}+$ $\cdots+s_{n_{i} i} x^{n_{i}} \quad 1 \leq i \leq t$.

It is easy to see that (1.1) is equivalent to the following formula (3.1)

$$
\left\{\begin{array}{l}
\sigma(x) S_{1}(x) \equiv r_{1}(x) \bmod x^{n_{1}+1} \\
\sigma(x) S_{2}(x) \equiv r_{2}(x) \bmod x^{n_{2}+1} \\
\cdots \\
\sigma(x) S_{t}(x) \equiv r_{t}(x) \bmod x^{n_{t}+1} \\
\text { for some polynomials } r_{i}(x) \text { such that } \operatorname{deg} r_{i} \leq l-1, \quad 1 \leq i \leq t .
\end{array}\right.
$$

Now, we set $f(x)=x^{l} \sigma\left(x^{-1}\right)=x^{l}+\sigma_{1} x^{l-1}+\sigma_{2} x^{l-2}+\cdots+\sigma_{l-1} x+\sigma_{l}$ and $S_{i}^{*}(x)=x^{n_{i}} S_{i}\left(x^{-1}\right)=s_{0 i} x^{n_{i}}+s_{1 i} x^{n_{i}-1}+\cdots+s_{n_{i} i}, 1 \leq i \leq t$. 
Hence, it is easily to see that (3.1) is equivalent to the following formula (3.2)

$$
\left\{\begin{array}{l}
f(x) S_{1}^{*}(x) \equiv a_{1}(x) \bmod x^{n_{1}+1} \\
f(x) S_{2}^{*}(x) \equiv a_{2}(x) \bmod x^{n_{2}+1} \\
\cdots \\
f(x) S_{t}^{*}(x) \equiv a_{t}(x) \bmod x^{n_{t}+1} \\
\text { for some polynomials } a_{i}(x) \text { such that } \operatorname{deg} a_{i} \leq l-1, \quad 1 \leq i \leq t .
\end{array}\right.
$$

Thus, finding linear feedback shift register of least length which generate sequences $s_{1}, \cdots, s_{t}$. is equivalent to find the polynomial $f(x)$ of least degree satisfying (3.2).

Once we find a polynomial $f(x)$ satisfying (3.2), then $\sigma(x)=x^{l} f\left(x^{-1}\right)$ is the polynomial satisfying (3.1), here $l=\operatorname{deg} f(x)$.

So, solving synthesis problem is equivalent to solving the following congruence equation group.

Finding $\left(a_{1}(x), a_{2}(x), \cdots, a_{t}(x), f(x)\right)$ such that

$$
\left\{\begin{array}{l}
f(x) S_{1}^{*}(x) \equiv a_{1}(x) \bmod x^{n_{1}+1} \\
f(x) S_{2}^{*}(x) \equiv a_{2}(x) \bmod x^{n_{2}+1} \\
\cdots \\
f(x) S_{t}^{*}(x) \equiv a_{t}(x) \bmod x^{n_{t}+1} \\
\operatorname{deg} a_{i}(x)<\operatorname{deg} f(x), \quad 1 \leq i \leq t \\
\min (\operatorname{deg} f(x))
\end{array}\right.
$$

Let $K=\left\{\left(a_{1}(x), \cdots, a_{t}(x), f(x)\right) \in F[x]^{t+1} \mid f(x) S_{i}^{*}(x) \equiv a_{i}(x) \bmod x^{n_{i}+1}\right.$, $1 \leq i \leq t\}$. Then we have the following lemma 3.1.

Lemma 3.1 $K$ is a submodule of free module $F[x]^{t+1}$ over polynomial ring $F[x]$.

Let $e_{1}=(1,0, \cdots, 0), e_{2}=(0,1,0, \cdots, 0), \cdots, e_{t+1}=(0,0, \cdots, 0,1)$ be the standard basis elements of $F[x]^{t+1}$, Namely,

$$
F[x]^{t+1}=\oplus_{i=1}^{t+1} F[x] e_{i} .
$$

Now, we have the following lemma 3.2

Lemma 3.2 Submodule $K$ is generated by $S_{1}^{*}(x) e_{1}+\cdots+S_{t}^{*}(x) e_{t}+$ $e_{t+1}, x^{n_{1}+1} e_{1}, \cdots, x^{n_{t}+1} e_{t}$.

Proof Let $\left(a_{1}(x), \cdots, a_{t}(x), f(x)\right) \in K$, then there exist $q_{1}(x), \cdots$, $q_{t}(x) \in F[x]$ such that $f(x) S_{i}^{*}(x)=a_{i}(x)+q_{i}(x) x^{n_{i}+1}, 1 \leq i \leq t$. Hence

$$
\begin{aligned}
\left(a_{1}(x), \cdots, a_{t}(x), f(x)\right)= & a_{1}(x) e_{1}+\cdots+a_{t}(x) e_{t}+f(x) e_{t+1} \\
= & \left(f(x) S_{1}^{*}(x)-q_{1}(x) x^{n_{1}+1}\right) e_{1} \\
& +\cdots \\
& +\left(f(x) S_{t}^{*}(x)-q_{t}(x) x^{n_{t}+1} e_{t}+f(x) e_{t+1}\right. \\
= & f(x)\left(S_{1}^{*}(x) e_{1}+\cdots+S_{t}^{*}(x) e_{t}+e_{t+1}\right. \\
& -q_{1}(x)\left(x^{n_{1}+1} e_{1}\right)-\cdots-q_{t}(x)\left(x^{n_{t}+1} e_{t}\right) .
\end{aligned}
$$

Hence, the lemma 3.2 is true. 
By lemma3.2, submodule $K$ has an explicit generators group. So, in order to solve problem (3.3), we consider suitable term order on monomial set of $F[x]^{t+1}$.

Now, monomial set of $F[x]^{t+1}$ is $\left\{x^{i} e_{j} \mid i \geq 0,1 \leq j \leq t+1\right\}$. We define a term order $>$ as follows:

$x^{i} e_{j}>x^{l} e_{j}$ iff $i>j$.

For $k<l, x^{i} e_{k}>x^{j} e_{l}$ iff $i \geq j$.

It is obvious that $>$ is a term order on monomial set of $F[x]^{t+1}$, and $e_{1}>e_{2}>\cdots>e_{t+1}$.

Now, we have the following lemma 3.3.

Lemma 3.3 Let $B$ be a minimal Grobner bases of $K$ with respect to the above term order $>$. Then $B$ must contain an element $\left(a_{1}(x), \cdots, a_{t}(x), f(x)\right)$ whose leading term involves in $e_{t+1}$, and $f(x)$ is a solution of (3.3).

By lemma 3.3, in order to solve problem (3.3), we only to compute a Grobner bases of $K$ with respect to $>$. As far as we know, there are at least 3 different algorithms to compute a Grobner bases of $K$.

For the convenience, we give two algorithms to solve this problem.

3.4. Buchberger algorithm.

Input: $F=\left(S_{1}^{*} e_{1}+\cdots+S_{t}^{*} e_{t}+e_{t+1}, x^{n_{1}+1} e_{1}, \cdots, x^{n_{t}+1} e_{t}\right)$, the above term order $>$.

Output: a Grobner bases $G$ for $M=\langle F\rangle$ with respect to $>$.

$G:=F$

REPEAT

$G^{\prime}:=G$

FOR each pair $f \neq g$ in $G^{\prime}$ DO

$S:=\overline{S(f, g)} G^{\prime}$

IF $S \neq 0$ THEN $G:=G \cup\{S\}$

UNTIL $G=G^{\prime}$.

Once $G$ is obtained, we can get a minimal Grobner bases of $M=<$ $F>$ from $G$.

Example 3.5. Let $F=G L(2), s_{1}=10101101, s_{2}=01010100 . n_{1}=$ $7, n_{2}=7$.

Let $S_{1}^{*}=x^{7}+0 \cdot x^{6}+x^{5}+0 \cdot x^{4}+x^{3}+x^{2}+0 \cdot x+1=x^{7}+x^{5}+x^{3}+x^{2}+1$. $S_{2}^{*}=0 \cdot x^{7}+x^{6}+0 \cdot x^{5}+x^{4}+0 \cdot x^{3}+x^{2}+0 \cdot x+0=x^{6}+x^{4}+x^{2}$.

So, Finding linear feedback shift register of least length synthesizing $s_{1}, s_{2}$ is equavilent to finding a polynomial $f(x)$ of least degree such that for some polynomials $a_{1}(x), a_{2}(x)$, the following formuula is true.

$\left\{\begin{array}{l}f(x) S_{1}^{*}(x) \equiv a_{1}(x) \bmod x^{8} \\ f(x) S_{2}^{*}(x) \equiv a_{2}(x) \bmod x^{8} \\ \operatorname{deg} f(x)<\operatorname{deg} a_{1}(x) \\ \operatorname{deg} f(x)<\operatorname{deg} a_{2}(x)\end{array}\right.$ 
Let $K=\left\{\left(a_{1}(x), a_{2}(x), f(x)\right) \mid f(x) S_{i}^{*}(x) \equiv a_{i}(x) \bmod x^{8}, 1 \leq i \leq 2\right\}$. then submodule $K$ of $F[x]^{3}$ has generators $S_{1}^{*} e_{1}+S_{2}^{*} e_{2}+e_{3}, x^{8} e_{1}, x^{8} e_{2}$, by lemma 3.2 .

Let term order $>$ be defined as follows:

(1) $x^{i} e_{k}>x^{j} e_{k}$ iff $i>j$.

(2) For $k<l, x^{i} e_{k}>x^{j} e_{l}$ iff $i \geq j$.

To solve synthesis problem for $s_{1}, s_{2}$, we need to find a minimal Grobner bases of $K$ with respect to the term order $>$, by lemma 3.3. In the following, we make use of Bucherberg algorithm to find a minimal Grobner bases of $K$ with respect to $>$.

Let $F=\left\{S_{1}^{*} e_{1}+S_{2}^{*} e_{2}+e_{3}, x^{8} e_{1}, x^{8} e_{2}\right\}$.

The first step. Let $f_{1}=S_{1}^{*} e_{1}+S_{2}^{*}+e_{3}, f_{2}=x^{8} e_{1}, f_{3}=x^{8} e_{2}$. Then $\operatorname{LT}\left(\mathrm{f}_{1}\right)=\mathrm{x}^{7} \mathrm{e}_{1}, \mathrm{LT}\left(\mathrm{f}_{2}\right)=\mathrm{x}^{8} \mathrm{e}_{1}, \mathrm{LT}\left(\mathrm{f}_{3}\right)=\mathrm{x}^{8} \mathrm{e}_{2}, \mathrm{G}:=\mathrm{F}$.

$S\left(f_{1}, f_{2}\right)=f_{2}-\frac{\mathrm{LT}\left(f_{2}\right)}{\operatorname{LT}\left(f_{1}\right)} f_{1}=\left(x^{6}+x^{4}+x^{3}+x, x^{7}+x^{5}+x^{3}, x\right)$.

$A_{1}={\overline{S\left(f_{1}, f_{2}\right)}}^{G}=\left(x^{6}+x^{4}+x^{3}+x, x^{7}+x^{5}+x^{3}, x\right)$.

$G:=\left\{f_{1}, f_{2}, f_{3}, A_{1}\right)$. $\mathrm{x}^{7} \mathrm{e}_{2}$.

The secend step. $\operatorname{LT}\left(f_{1}\right)=x^{7} e_{1}, \operatorname{LT}\left(f_{2}\right)=x^{8} e_{1}, \operatorname{LT}\left(f_{3}\right)=x^{8} e_{2}, \operatorname{LT}\left(A_{1}\right)=$

$S\left(f_{3}, A_{1}\right)=f_{3}-\frac{\mathrm{LT}\left(\mathrm{f}_{3}\right)}{\mathrm{LT}\left(\mathrm{A}_{1}\right)} A_{1}=\left(x^{7}+x^{5}+x^{4}+x^{2}, x^{6}+x^{4}, x^{2}\right)$.

$A_{2}=\overline{S\left(f_{3}, A_{1}\right)}{ }^{G}=\left(x^{4}+x^{3}+1, x^{2}, x^{2}+1\right)$.

$G:=\left\{f_{1}, f_{2}, f_{3}, A_{1}, A_{2}\right\}$.

The third step. $\operatorname{LT}\left(\mathrm{f}_{1}\right)=\mathrm{x}^{7} \mathrm{e}_{1}, \operatorname{LT}\left(\mathrm{f}_{2}\right)=\mathrm{x}^{8} \mathrm{e}_{1}, \operatorname{LT}\left(\mathrm{f}_{3}\right)=\mathrm{x}^{8} \mathrm{e}_{2}$,

$\operatorname{LT}\left(\mathrm{A}_{1}\right)=\mathrm{x}^{7} \mathrm{e}_{2}, \operatorname{LT}\left(\mathrm{A}_{2}\right)=\mathrm{x}^{4} \mathrm{e}_{2}$.

$S\left(f_{1}, A_{2}\right)=f_{1}-\frac{\mathrm{LT}\left(\mathrm{f}_{1}\right)}{\mathrm{LT}\left(\mathrm{A}_{2}\right)} A_{2}=\left(x^{6}+x^{5}+x^{2}+1, x^{6}+x^{5}+x^{4}+x^{2}, x^{5}+\right.$ $\left.x^{3}+1\right)$.

$A_{3}={\overline{S\left(f_{1}, A_{2}\right)}}^{G}=S\left(f_{1}, A_{2}\right)-\frac{x^{6} e_{1}}{x^{4} e_{1}} A_{2}=\left(1, x^{6}+x^{5}+x^{2}, x^{5}+x^{4}+\right.$ $\left.x^{3}+x^{2}+1\right)$.

$G:=\left\{f_{1}, f_{2}, f_{3}, A_{1}, A_{2}, A_{3}\right\}$.

The fourth step. $\operatorname{LT}\left(\mathrm{A}_{1}\right)=\mathrm{x}^{7} \mathrm{e}_{2}, \operatorname{LT}\left(\mathrm{A}_{2}\right)=\mathrm{x}^{4} \mathrm{e}_{1}, \operatorname{LT}\left(\mathrm{A}_{3}\right)=\mathrm{x}^{6} \mathrm{e}_{2}$.

$S\left(A_{1}, A_{3}\right)=A_{1}-\frac{\operatorname{LT}\left(A_{1}\right)}{\operatorname{LT}\left(A_{3}\right)} A_{3}=\left(x^{6}+x^{4}+x^{3}, x^{6}+x^{5}, x^{6}+x^{5}+x^{4}+x^{3}\right)$.

Let $A_{4}=\overline{S\left(A_{1}, A_{3}\right)}$. Then

$A_{4}^{\prime}=S\left(A_{1}, A_{3}\right)-\frac{x^{6} e_{1}}{x^{4} e_{1}} A_{2}=\left(x^{5}+x^{4}+x^{3}+x^{2}, x^{6}+x^{5}+x^{4}, x^{6}+x^{5}+\right.$ $\left.x^{3}+x^{2}\right)$.

$A_{4}^{\prime \prime}=A_{4}^{\prime}-\frac{x^{5} e_{1}}{x^{4} e_{1}} A_{2}=\left(x^{3}+x^{2}+x, x^{6}+x^{5}+x^{4}+x^{3}, x^{6}+x^{5}+x^{2}+x\right)$.
$A_{4}=A_{4}^{\prime \prime}-\frac{x^{6} e_{2}}{x^{6} e_{2}} A_{3}=\left(x^{3}+x^{2}+x+1, x^{4}+x^{3}+x^{2}, x^{6}+x^{4}+x^{3}+x+1\right)$.

We note that $\operatorname{LT}\left(\mathrm{A}_{4}\right)=\mathrm{x}^{6} \mathrm{e}_{3}$.

$G=\left\{f_{1}, f_{2}, f_{3}, A_{1}, A_{2}, A_{3}, A_{4}\right\}$. is a Grobner bases of $M=<F>$ with repect to $>$, and $\left\{A_{2}, A_{3}, A_{4}\right\}$ is a minimal Grobner bases of $M=<F>$ 
with respect to $>$. The least length of linear feedback shift register synthesizing $s_{1}, s_{2}$ is 6 .

The characteristic polynomial of the LFSR is $f(x)=x^{6}+x^{4}+x^{3}+x+1$. and the corresponding LFSR is (in polynomial form) $\sigma(x)=x^{6} f(x)=$ $x^{6}\left(x^{-6}+x^{-4}+x^{-3}+x^{-1}+1\right)=x^{6}+x^{5}+x^{3}+x^{2}+1$.

All the characteristic polynomials of least degree can be given in the set

$\left\{f(x)=g_{1}(x)\left(x^{6}+x^{4}+x^{3}+x+1\right)+g_{2}(x)\left(x^{5}+x^{4}+x^{3}+x^{2}+1\right)+\right.$ $\left.g_{3}(x)\left(x^{2}+1\right) \mid g_{i}(x) \in F[x], 1 \leq i \leq 3, \operatorname{deg} f(x)=6\right\}$.

3.6. FGLM algorithm.

Let $r=\max \left(\operatorname{deg} S_{i}^{*}(x)\right)$. We define a term order $>_{1}$ on monomial set of $F[x]^{t+1}$ as follows:

(1) $x^{i} e_{j}>_{1} x^{l} e_{j}$ iff $i>l$.

(2) For $k, l=1, \cdots, t$ and $k<l, x^{i} e_{k}>_{1} x^{j} e_{l}$ iff $i \geq j$.

(3) For $j=1, \cdots, t, x^{a} e_{t+1}>_{1} x^{b} e_{j}$ iff $a+r \geq b$.

It is easily to see that $>_{1}$ is a term order and $\left\{f_{1}=x^{n_{1}+1} e_{1}, \cdots, f_{t}=\right.$ $x^{n_{t}+1} e_{t}$,

$\left.f_{t+1}=S_{1}^{*} e_{1}+\cdots+S_{t}^{*} e_{t}+e_{t+1}\right\}$ is a Grobner bases of $K$ with respect to $>_{1}$.

Our problem is to find a Grobner bases of $K$ with respect to $>$. So we make use of FGLM algorithm to convert the above Grobner bases of $K$ with respect to $>_{1}$ into a Grobner bases of $K$ with respect to $>$.

For a given zero-dimensional ideal I, by FGLM algorithm, a Grobner bases of I with respect to a monomial order can be convert to a Grobner bases of I with repect to another monomial order. For FGLM algorithm, we refer to [8], [4], [6].

In [8], FGLM algorithm was extend to zero-dimensional submodule case. Actually, FGLM algorithm for sumodules is essentially same as FGLM algorithm for ideals. In the following, we outline FGLM algorithm as follows: (Here, we only consider Grobner bases in $F[x]^{t+1}$.)

FGLM algorithm.

Input: $G=\left\{f_{1}, f_{2}, \cdots, f_{t}, f_{t+1}\right\}$, the monomial order $>$.

Output: a Grobner bases $G_{>}$with repect to $>$, a list $B_{>}$.

(1) Main step. Given the input $x^{\alpha} e_{i}$, compute $\overline{x^{\alpha} e_{i}} G$.

(a) If $\overline{x^{\alpha} e_{i}} G$ is linearly dependent on the remainders (on divisin by $G$ ) of the monomials in $B_{>}$, then we have a linear combination:

$$
{\overline{x^{\alpha} e_{i}}}^{G}-\sum_{j_{k}} c_{j_{k}}{\overline{x^{\alpha\left(j_{k}\right)} e_{k}}}^{G}=0 .
$$

Where $x^{\alpha\left(j_{k}\right)} e_{k} \in B_{>}$and $c_{j_{k}} \in F$.

In this case, we add $g=x^{\alpha} e_{i}-\sum c_{j_{k}} x^{\alpha\left(j_{k}\right)} e_{k}$ to $G_{>}$. 
(b) If ${\overline{x^{\alpha}}}_{i}$ G is linearly independent from the remainders (on division by $G$ ) of the monomials in $B_{>}$, the we add $x^{\alpha} e_{i}$ to $B_{>}$.

(2) Next monomial. Relace $x^{\alpha} e_{i}$ with the next monomial in $>$ which is not divisible by any of the $\operatorname{LT}\left(g_{i}\right), g_{i} \in G_{>}$.

(3) Termination Test. If the Main loop added a polynomial $g$ to $G_{>}$, then compute LT(g). If for every $e_{i}$, we have an element $g \in G_{>}$auch that $\operatorname{LT}\left(\mathrm{g}_{\mathrm{i}}\right)$ involves in $e_{i}$, then the algorithm Terminates.

Example 3.7 Let $F=G L(2), s_{1}=10101101, s_{2}=01010100, n_{1}=$ $7, n_{2}=7$.

Let $S_{1}^{*}(x), S_{2}^{*}(x)$ and $K$ as in example 2.4. With the definition of $>_{1}$, $G=\left\{f_{1}=x^{8} e_{1}, f_{2}=x^{8} e_{2}, f_{3}=S_{1}^{*} e_{1}+S_{2}^{*} e_{2}+e_{3}\right\}$ is a Grobner bases of $K$ with respect to $>_{1}$. We use FGLM algorithm to compute a minimal Grobner bases of $K$ with respect to the monomial order $>$.

We order monomials by $>$ as follows:

$e_{3}<e_{2}<e_{1}<x e_{3}<x e_{2}<x e_{1}<x^{2} e_{3}<x^{2} e_{2}<x^{2} e_{1}<x^{3} e_{3}<x^{3}$

$e_{2}<x^{3} e_{1}<x^{4} e_{3}<\cdots$

We have the following computation steps:

1. ${\overline{e_{3}}}^{G}=\left(x^{7}+x^{5}+x^{3}+x^{2}+1\right)+\left(x^{6}+x^{4}+x^{2}\right) e_{2}$.

2. ${\overline{e_{2}}}^{G}=e_{2}$.

3. $\overline{e_{1}} G=e_{1}$.

4. $\overline{x e_{3}} G=\left(x^{6}+x^{4}+x^{3}+x\right) e_{1}+\left(x^{7}+x^{5}+x^{3}\right) e_{2}$.

5. $\overline{x e_{2}} G=x e_{2}$.

6. $\overline{x e_{1}} G=x e_{1}$.

7. $\overline{x^{2} e_{3}}=\left(x^{7}+x^{5}+x^{4}+x^{2}\right) e_{1}+\left(x^{6}+x^{4}\right) e_{2}$.

8. $\overline{x^{2} e_{2}}=x^{2} e_{2}$.

9. $\overline{x^{2} e_{1}}=x^{2} e_{1}$.

10. ${\overline{x^{3} e_{3}}}^{G}=\left(x^{6}+x^{5}+x^{3}\right) e_{1}+\left(x^{7}+x^{5}\right) e_{2}$.

11. $\overline{x^{3} e_{2}}=x^{3} e_{2}$.

12. $\overline{x^{3} e_{1}} G=x^{3} e_{1}$.

13. ${\overline{x^{4} e_{3}}}^{G}=\left(x^{7}+x^{6}+x^{4}\right) e_{1}+x^{6} e_{2}$.

14. $\overline{x^{4} e_{2}} G=x^{4} e_{2}$.

15. $\overline{x^{4} e_{1}} G=x^{4} e_{1}$.

In this case, ${\overline{x^{4} e_{1}}}^{G}={\overline{e_{3}}}^{G}+{\overline{x^{2} e_{3}}}^{G}+{\overline{x^{2} e_{2}}}^{G}+{\overline{x^{3} e_{1}}}^{G}+{\overline{e_{1}}}^{G}$.

So, $x^{4} e_{1}+e_{1}+x^{3} e_{1}+x^{2} e_{2}+e_{3}+x^{2} e_{3} \in G_{>}$.

16. $\overline{x^{5} e_{3}}=\left(x^{7}+x^{5}\right) e_{1}+x^{7} e_{2}$.

17. $\overline{x^{5} e_{2}}=x^{5} e_{2}$.

18. $\overline{x^{6} e_{3}}=x^{6} e_{1}$.

In this case, ${\overline{x^{2} e_{1}}}^{G}+{\overline{e_{1}}}^{G}+{\overline{x^{4} e_{2}}}^{G}+{\overline{x^{3} e_{2}}}^{G}+{\overline{x^{2} e_{2}}}^{G}+{\overline{x^{6} e_{3}}}^{G}+{\overline{x^{4} e_{3}}}^{G}+$ ${\overline{x^{3} e_{3}}}^{G}+\overline{x e_{3}}{ }^{G}+\overline{e_{3}} G=0$. 
So, $\left(x^{3}+x^{2}+x+1, x^{4}+x^{3}+x^{2}, x^{6}+x^{4}+x^{3}+x+1\right) \in G_{>}$.

19. ${\overline{x^{6} e_{2}}}^{G}=x^{6} e_{2}$. 0 .

In this case, ${\overline{x^{4} e_{1}}}^{G}+{\overline{x^{3} e_{1}}}^{G}+{\overline{x^{6} e_{2}}}^{G}+{\overline{x^{5} e_{2}}}^{G}+{\overline{x^{5} e_{3}}}^{G}+{\overline{x^{4} e_{3}}}^{G}+{\overline{x^{3} e_{3}}}^{G}=$

So, $\left(x^{4}+x^{3}, x^{6}+x^{5}, x^{5}+x^{4}+x^{3}\right) \in G_{>}$.

Thus, we get a minimal Grobner bases of $K$ as follows:

$G_{>}=\left\{g_{1}=\left(x^{4}+x^{3}+1, x^{2}, x^{2}+1\right), g_{2}=\left(x^{4}+x^{3}, x^{6}+x^{5}, x^{5}+x^{4}+\right.\right.$ $\left.\left.x^{3}\right), g_{3}=\left(x^{3}+x^{2}+x+1, x^{4}+x^{3}+x^{2}, x^{6}+x^{4}+x^{3}+x+1\right)\right\}$.

We have $\operatorname{LT}\left(\mathrm{g}_{1}\right)=\mathrm{x}^{4} \mathrm{e}_{1}, \operatorname{LT}\left(\mathrm{g}_{2}\right)=\mathrm{x}^{6} \mathrm{e}_{2}, \operatorname{LT}\left(\mathrm{g}_{3}\right)=\mathrm{x}^{6} \mathrm{e}_{3}$. and $\operatorname{LT}\left(\mathrm{g}_{2}\right)>$ $\operatorname{LT}\left(\mathrm{g}_{3}\right)>\operatorname{LT}\left(\mathrm{g}_{1}\right)$.

Let us give the following definition ([9]).

Definition 3.8 Let $K$ be a non-zero submodule and $>$ be a monomial order. we say that $f \in K$ is a minimal element of $K$ with respect to $>$ if $\operatorname{LT}(\mathrm{f})$ is a minimal element of $\mathrm{LT}(\mathrm{K})$ with respect to $>$, where we denote by LT(K) the set of leading monomials of elements of $K$.

When $t=1$, from [9], the solutions of (3.3) are minimal elements of $K$. But, when $t>1$, from example (3.7), we see that the solutions of $K$ are not minimal elements.

\section{References}

[1] L. Althaler and A. Dur. A generalization of Massey-Ding algorithm. AAECC 9, (1998).

[2] L. Althaler and A. Dur. Finite Linear Recurring Sequences and Homogeneous Ideals. AAECC 7, 377-390 (1996).

[3] W. Adams and P. Loustaunau. A Introduction to Grobner Bases, AMs, Providence RI, 1994.

[4] T. Becker and V. Weispenning. Grobner Bases, Springer-Verlag, New York, 1993.

[5] D. Cox, J. Little and D. O'SHea. Ideals, Varieties, and Algorithm, Springer-Verlag, New York, 1996.

[6] D. Cox, J. Little and D. O'Shea. Using Algebraic Geometry, Springer-Verlag, New York, 1998.

[7] D. Eisenbud. Commutative Algebra with a View Toward Algebraic Geometry, Springer-Verlag, New York, 1995.

[8] P. Fitzpatrick. Solving a multivariable congruence by change of term order (1997) 24, 575-589.

[9] P. Fitzpatrick. On the key equation, IEEE Trans. on Information Theory. 41 (1995), 1290-1302. 
[10] G. Feng and K. Tzeng. A generalized Euclidean algorithm for multisequence shift-register synthesis. IEEE Trans. on Inform theory, (1989), 35(3), 584-594. 